\title{
Towards an Affordable Real-Time Cardiovascular Emergency Detection and Response System
}

\author{
Ahmed EIShafee \\ Assistant Professor \\ Ahram Canadian University \\ 6th October City, \\ Giza, Egypt
}

\begin{abstract}
Cardiovascular diseases are always accompanied with critical symptoms that can cause sudden death. Those symptoms may not be recognized by most of humans. So it could be too late when those symptoms are recognized and assigned to medical help. Pervasive health care systems can solve this dilemma by continuous monitoring of suspected patients. Pervasive health care is used to shift the already established, hospital based, centralized health care system to another ubiquitous, usercentered, and mobile system as an overall preventive health management system. Pervasive health care is expected to have a strong impact on quality and efficiency of health care domain as well as a strong impact on patients' quality of life. Because of its ubiquitous, autonomous nature, it also helps to increase independency and emergency prevention. This paper presents an overview of recently published technical researches regarding the pervasive health care as an application of ubiquitous computing. As well as introducing the authors' new model and prototype for a new proposed system of an affordable real time emergency detection and response of critical heart conditions Aff-HRM that is especially designed for financially not-capable cardio patients.
\end{abstract}

\section{Keywords}

Ubiquitous health monitoring, ECG detection, pervasive healthcare, Cardiovascular.

\section{INTRODUCTION}

Continuous advances of medical technologies and methodologies, helped in the noticeable extension of human average living age, which in turn increased the pressure of aging population on healthcare systems. Also increased the financial support afforded by governmental organization to comply with the requiring health care insurance sector. Elderly are more likely to suffer from health conditions which can prejudice their independent living.

Pervasive health care is the applying of pervasive computing principles in health care domain. Or it is the delivery of health related services and information via ubiquitous computing technologies.

Pervasive health care is used to shift the already established, hospital based, centralized health care system to another ubiquitous, user-centered, and mobile system as an overall preventive health management system [1] [2].

Ubiquitous computing is applied on a wide range of applications and domains, like distributed systems, mobile computing, sensors networks, HCI, context awareness, and artificial intelligent. Combined knowledge from all these domains and disciplines, is necessary to implement a reliable ubiquitous system. In pervasive health care integrating knowledge from medical disciplines like, medicine, physiotherapy, psychology, and information technology is essential in implementing a reliable pervasive health care system.

Pervasive health care is expected to have a strong impact on quality and efficiency of health care domain as well as a strong impact on patients' quality of life. Because of its ubiquitous, autonomous nature, it also helps to increase independency and emergency prevention.

In pervasive health care domain, human is integrated into the network and considered as a part of it. This can be done by equipping the human with one the well-known communication technologies like wearable RFID tags, or microelectronic sensors. RFID is used for automatic identification and data capture using radio frequency transmission. It transfers collected data between the object with tag and the reader, to facilitate identification, tracking of objects [3].

Low power, inexpensive sensors and monitoring devices with large storage scale are embedded in ubiquitous health care domain in order to collect data required for an effective operation of pervasive health care system. Fig. 1 Shows pervasive health care model using ubiquitous computing principles [1]. Fig. 2 shows the standardization of human vital signals digitization.

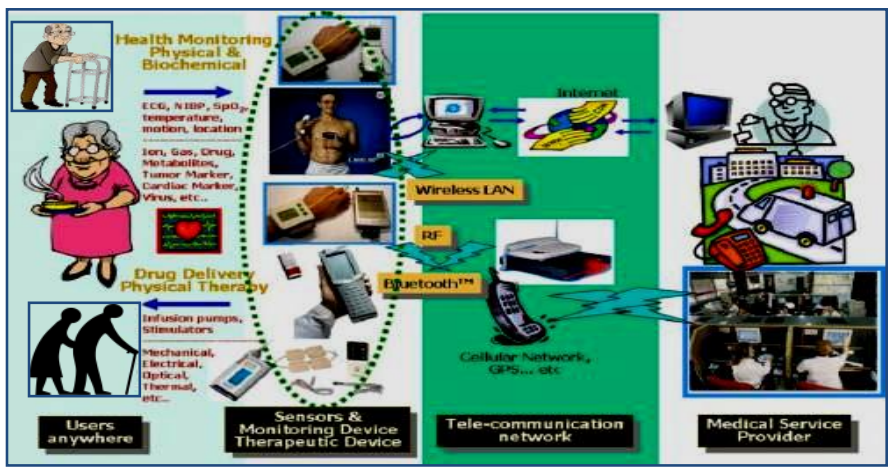

Fig 1: Pervasive health care model using ubiquitous computing principles. 


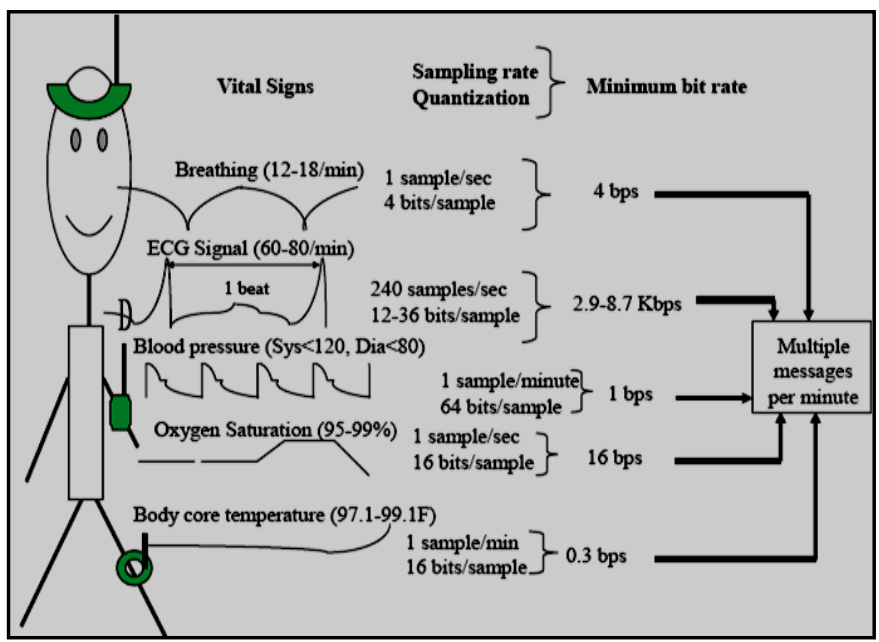

Fig 2: Human Vital Signals Digitaizatin Standard Model.

The authors conducted this research in order to present a survey on some of recently published technical researches regarding the pervasive health care as an application of ubiquitous computing.

As well as introducing their own model regarding a prototype for a new proposed system of an affordable real-time emergency detection and response of critical heart conditions Aff-HRM. The proposed system is consisting of many software/hardware components. Two software components using Client/Server Approach are implemented. A wearable Bluetooth enabled heart beat (ECG) monitoring sensor is used as the monitoring middleware. Bluetooth, WLAN enabled windows or android based smart phone with the client software module installed. And finally an internet connection enabled server computer system which is prepared to respond to critical heart condition of a certain user according to the data received through the WAN.

The remaining of this paper is organized as follows: section II is summarizing some published researches of pervasive health care computing systems. The conducted survey provides an overview of recent development and implementation. Technologies used, pros and cons of the systems are the main concern of the author's survey. Section III is presenting Pervasive health care applications and general requirements of pervasive health care systems. A proposal for the new realtime emergency detection and response system of critical heart conditions will be presented in section IV. Finally challenges facing pervasive healthcare systems implementation and deployment will be summarized in section $\mathrm{V}$.

\section{LITERATURE REVIEW}

The rapid development of ubiquitous computing concepts is increasingly affecting health care systems. This is encouraging researches to widely investigate the new capabilities and technologies. Authors summarize some of these recent researches and highlight some of the experiences reported in deployment processes. Most of the researches are in their prototype stages, case studies, or pilot studies [4].

Starting with, Diletta Cacciagrano, et al. [2] they introduced a three layers methodological approach for a personalized and ubiquitous health care system that can provide autonomous and run-time response. Application layer is responsible for data gathering via environmental sensors. Then this information is managed and organized in the logic layer.
Finally organized information is processed and elaborated in physical layer.

Static sensor networks are embedded in local smart space as well as RFID tags are attached either to objects or people. Together plus some social information about the user -can be extracted from user's social account like face book or twitter) - they can fully characterize the individual context from the authors' point of view.

The proposed system should automatically take the correct actions based on the knowledge of what is happening in the patient's environment. The system has been partially tested in health care domain for the development of drug dispensary services for people with mild cognitive impairments.

Actually from our point of view the system capabilities are very limited, and it requires much more development efforts to reach what Diletta Cacciagrano, et al. are trying to do.

The second research is done by Junho Moon and Dongsoo Kim [5]. They proposed a context-aware business process management for personalized health care services. The proposed system is an integrated architecture of contextawareness BPM based on ubiquitous computing. It is hospital based and it helps the health care professionals to decide the most appropriate medical treatment services according to each patient status but it is supporting the ubiquitous services concept.

The patient's status is collected from ubiquitous data sources in addition to the electronic medical record (EMR) located on hospital's database. Ubiquitous personalized data sources are such as RFID and smart sensors. The proposed system is supposed to improve the personalized clinical treatment services. The system architecture is shown in Fig. 3. [5]

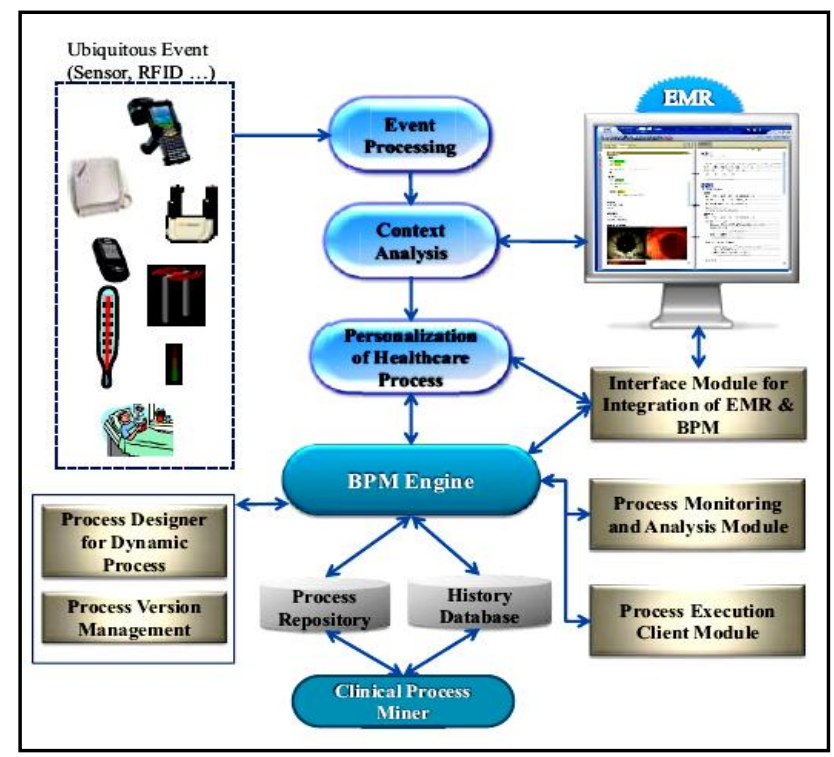

Fig 3: System architecture for personalized healthcare services [5].

The third paper is done by Garth V. Crosby, et al [6]. It introduces a survey for using wireless body area networks (WBANs) in ubiquitous health care systems. This research is very precious as it covers monitoring and sensing in WBANs, an examination of power efficient protocols, the WBAN system architectures, Approaches to routing in WBAN, and finally various security techniques and protocols are presented.

For the monitoring and sensing in WBANs, we summarized their findings in the following table. Table I summarizes 
WBAN sensors and monitoring devices as per Garth V. Crosby, et al [6].

The authors also discussed the most power efficient protocols and they decided that DQBAN (Distributed Queuing Body Area Network) for WBAN introduced by Otal and Alonso is the most appropriate protocol among other protocols that were discussed in the research. DQBAN allows simplified routing, lower interference and idle listening; all of which contribute to energy-efficiency for body area networks.

Then the research classified the WBAN topology architecture to two categories; Flat, and Muli-tier as shown in Fig. 4.
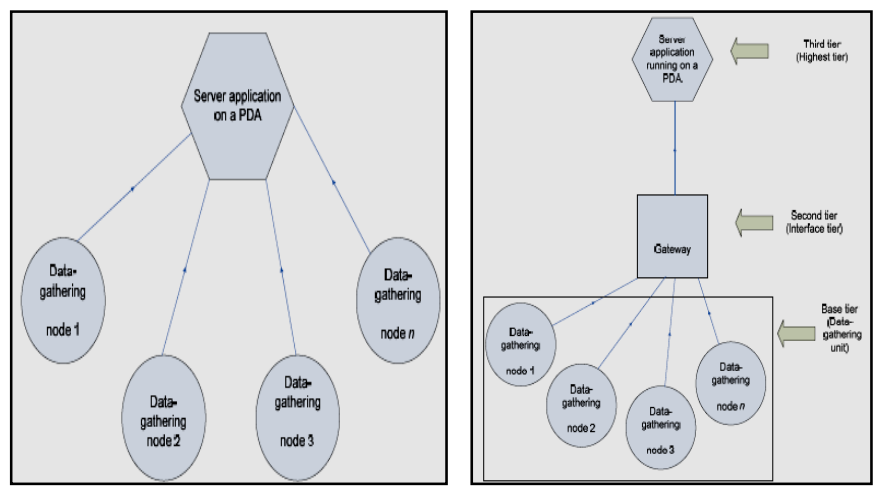

Fig 4: Flat and Multi-tier architecture for WBANs [6].

For the most optimum routing protocol that suits WBANs, after reviewing all proposed protocol in previously published researches, the authors introduced a possible approach of using temporal mobility patterns of nodes based on historical data of link disconnections.

A temporal pattern of link disconnection data can be used to select links for computing a probabilistic end-to-end path. However, extensive evaluations of these protocols need to be conducted based on prototype implementation in laboratories [6].

And finally they discussed the security requirements of WBANs. WBANs infrastructure must implement security operations that guarantee the security, data integrity, privacy and confidentiality of the patients' medical records as well as the availability of services.

Table 1. WBAN Sensors and monitoring devices

\begin{tabular}{|c|c|c|c|}
\hline $\begin{array}{c}\text { Sensor } \\
\text { Type }\end{array}$ & Example & Function & Picture \\
\hline \multirow{3}{*}{$\begin{array}{l}\text { Wearab } \\
\text {-le } \\
\text { sensors }\end{array}$} & $\begin{array}{l}\text { Pulse } \\
\text { Oximetry }\end{array}$ & $\begin{array}{l}\text { measures the } \\
\text { oxygen saturation } \\
\text { levels in blood as } \\
\text { well as the } \\
\text { changes in blood } \\
\text { volume in the skin } \\
\text { that coincide with } \\
\text { the cardiac cycle. }\end{array}$ & \\
\hline & $\begin{array}{l}\text { Electro- } \\
\text { cardiograp } \\
\text {-hy (ECG) }\end{array}$ & $\begin{array}{l}\text { ECG is a } \\
\text { waveform that } \\
\text { represents the } \\
\text { propagation of } \\
\text { electric potentials } \\
\text { through the heart } \\
\text { muscle with } \\
\text { respect to time. }\end{array}$ & 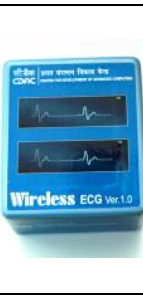 \\
\hline & Blood & A blood pressure & \\
\hline
\end{tabular}

\begin{tabular}{|c|c|c|c|}
\hline & Pressure & $\begin{array}{l}\text { (BP) reading is a } \\
\text { measure of the } \\
\text { force exerted by } \\
\text { circulating blood } \\
\text { on the walls of } \\
\text { blood vessels. BP } \\
\text { varies between a } \\
\text { maximum } \\
\text { (systolic) and a } \\
\text { minimum } \\
\text { (diastolic) } \\
\text { pressure during a } \\
\text { cardiac cycle. }\end{array}$ & 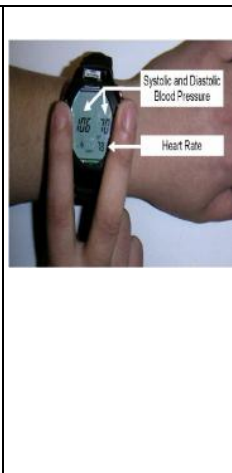 \\
\hline & $\begin{array}{l}\text { Electro- } \\
\text { myograph } \\
\text { y (EMG) }\end{array}$ & $\begin{array}{l}\text { Electromyography } \\
\text { is the study of } \\
\text { muscle function } \\
\text { through the } \\
\text { monitoring of the } \\
\text { electrical signals } \\
\text { emitted by the } \\
\text { muscle. }\end{array}$ & \\
\hline & $\begin{array}{l}\text { Activity/ } \\
\text { Motion } \\
\text { Detection } \\
\text { The level } \\
\text { of activity } \\
\text { or the } \\
\text { nature of } \\
\text { motion of } \\
\text { an } \\
\text { individual } \\
\text { can be } \\
\text { detected } \\
\text { by a } \\
\text { system } \\
\text { That } \\
\text { combines } \\
\text { an } \\
\text { accelero- } \\
\text { meter with } \\
\text { a } \\
\text { gyroscope }\end{array}$ & $\begin{array}{l}\text { An accelerometer } \\
\text { is a sensor that } \\
\text { measures } \\
\text { acceleration with } \\
\text { respect to gravity, } \\
\text { and can be used to } \\
\text { determine the } \\
\text { orientation of a } \\
\text { body part in the } \\
\text { absence of } \\
\text { movement. A } \\
\text { gyroscope is a } \\
\text { sensor that } \\
\text { measures angular } \\
\text { velocity and can } \\
\text { be used to } \\
\text { determine the } \\
\text { orientation of a } \\
\text { moving body part } \\
\text { as a function of } \\
\text { time. }\end{array}$ & \\
\hline \multirow[t]{2}{*}{$\begin{array}{l}\text { Implant } \\
\text { able } \\
\text { Sensors }\end{array}$} & $\begin{array}{l}\text { Glucose } \\
\text { Monitori- } \\
\text { ng }\end{array}$ & $\begin{array}{l}\text { real-time } \\
\text { continuous blood } \\
\text { glucose data is } \\
\text { recorded and sent } \\
\text { using RF } \\
\text { transmission } \\
\text { media. sensor is } \\
\text { implanted in the } \\
\text { subcutaneous } \\
\text { tissue of the } \\
\text { abdomen. }\end{array}$ & \\
\hline & $\begin{array}{l}\text { Implanta- } \\
\text { ble Neural } \\
\text { Stimulato- } \\
\text { rs }\end{array}$ & $\begin{array}{l}\text { It sends electrical } \\
\text { impulses into the } \\
\text { brain or spinal } \\
\text { cord for the } \\
\text { treatment of } \\
\text { Parkinson's } \\
\text { disease }\end{array}$ & 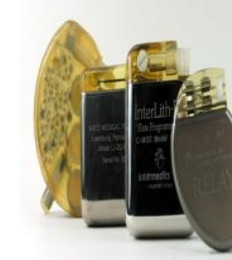 \\
\hline
\end{tabular}

The authors discussed many security solutions proposed in other researches. These solutions should be emerged in a WBAN to obtain the maximum security level required. 
TinySec is proposed by S.S.Marci, et al [7]. It is a security solution for biomedical sensor network to achieve link-layer encryption and data authentication. TinySec does not protect against node capture. If a node is compromised and keying material revealed the entire network can be compromised.

Table II. Shows several security suites can be implemented under the WBAN IEEE 802.15.4.

Table 2. WBAN IEEE 802.15.4 Security Suites

\begin{tabular}{|c|c|c|}
\hline Name & Description & Comment \\
\hline Null & No security & \\
\hline $\begin{array}{l}\text { AES-CTR } \\
\text { Counter Mode }\end{array}$ & $\begin{array}{l}\text { Encryption only. } \\
\text { This provides access } \\
\text { control, data encryption, } \\
\text { and optional sequential } \\
\text { freshness. }\end{array}$ & $\begin{array}{l}\text { Block cipher } \rightarrow \\
\text { Stream cipher } \\
\text { to secure stream } \\
\text { broadcasted data } \\
\text { (provide } \\
\text { encryption only) }\end{array}$ \\
\hline $\begin{array}{l}\text { AES-CBCMAC- } \\
128 \\
\text { AES-CBCMAC- } \\
64 \\
\text { AES-CBCMAC- } \\
32 \\
\text { AES with } \\
\text { operation modes: } \\
\text { Cipher block } \\
\text { chaining CBC } \\
\text { and message } \\
\text { authentication } \\
\text { code MAC }\end{array}$ & $\begin{array}{l}\text { Authentication only. } \\
\text { Allowing flexibility by } \\
\text { the selection of different } \\
\text { MAC lengths: } 32,64,128 \\
\text { bits. }\end{array}$ & $\begin{array}{l}\text { Block cipher } \rightarrow \\
\text { Stream cipher } \\
\text { to secure stream } \\
\text { broadcasted data } \\
\text { (provide } \\
\text { authentication } \\
\text { only) } \\
\text { CBC process the } \\
\text { plain text in series } \\
\text { (each block of } \\
\text { cipher is chained } \\
\text { with the key to } \\
\text { produce the next } \\
\text { block cipher and } \\
\text { so on) }\end{array}$ \\
\hline $\begin{array}{l}\text { AES-CCM- } 128 \\
\text { AES-CCM- } 64 \\
\text { AES-CCM- } 32 \\
\text { AES with } \\
\text { operation modes: } \\
\text { Counter mode } \\
\text { CTR message } \\
\text { And authentication } \\
\text { code MAC }\end{array}$ & $\begin{array}{l}\text { Authentication and } \\
\text { Encryption } \\
\text { allowing flexibility by the } \\
\text { selection of different } \\
\text { MAC lengths: 32, 64, } 128 \\
\text { bits }\end{array}$ & $\begin{array}{l}\text { Block cipher } \rightarrow \\
\text { Stream cipher } \\
\text { to secure stream } \\
\text { broadcasted data } \\
\text { (provide } \\
\text { encryption and } \\
\text { authentication) } \\
\text { CTR process the } \\
\text { plain text in } \\
\text { parallel mode } \\
\text { while each block } \\
\text { of plain text is } \\
\text { processed to } \\
\text { produce its cipher } \\
\text { and so on. }\end{array}$ \\
\hline
\end{tabular}

This research is very beneficial to all researchers in the sensor networks field especially, in the biomedical and health care domain.

The fourth research is done by Sanjay Bhadoria and Hitesh Gupta [8]. This research presents a review on mobile based health monitoring systems. It also discusses the desirable properties of a good mobile health care system as well as few existing health care applications [8]. Fig. 5 shows the typical architecture of mobile health care systems.

As per the research, the authors classified health care monitoring systems into two categories:

- Real-time health monitoring system:

- Constant transfer of individual biomedical data in real-time.
- Allow immediate response in case of emergencies.

- Require high communication and power cost.

- Store-and-forward health monitoring system:

- Record biomedical data and only transmit it on regular time bases.

- Unable to handle emergencies.

- May retain privacy, if the data is processed sufficiently.

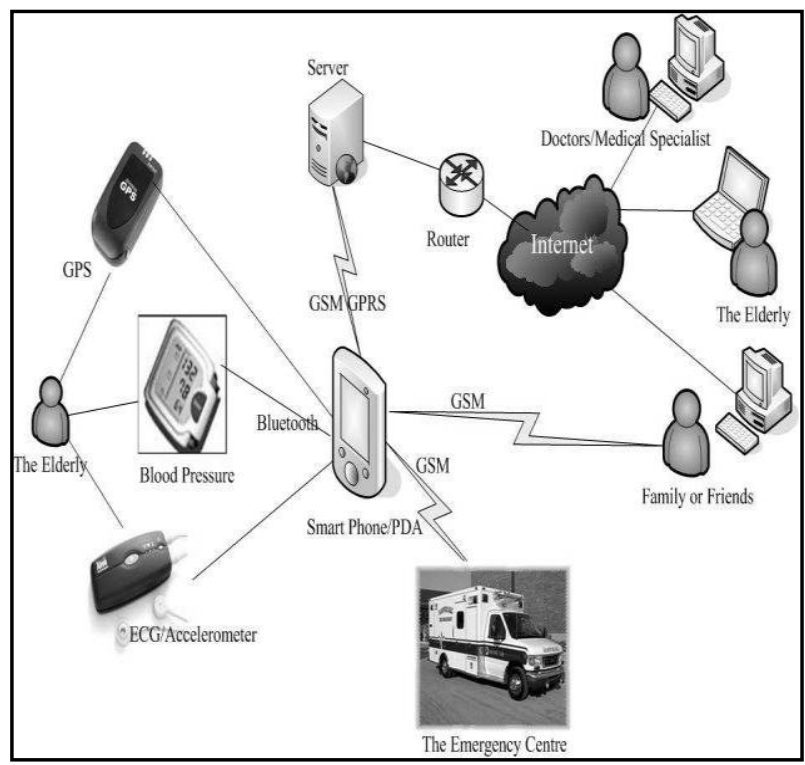

Fig 5: Typical architecture of mobile health care systems

[8]

The research summarized some of previously proposed health monitoring system using mobile devices. MobiHealth, WiMoca, MORF, and WAITER were discussed. The first three systems still present certain limitations related to the fact that the analysis is not performed in the place where the signal is acquired. WAITER is a relatively static monitoring system in which the status is set statically and doctors are called when mobile phone send alert messages. It is not sufficient in realtime and dynamic monitoring [8].

Then the research reviewed some of the well-known commercial applications for mobile medical care. AID-N is depending on wireless communication between the wearable mote and the nearest tablet or mobile phone in the range of its Ad Hoc wireless network. It measures HB, BP, SPO2, temp, Activity/motion, and location. The system is useful for patient monitoring and tracking in case of emergency.

AMON is a wearable (wrist-worn) medical monitoring system that uses a direct connection like (SMS) to send the collected data from the patient. Although the concept has been validated but some problems in system's functionality have raised during the testing phase of the prototype.

HealthGear is another system that was developed at MICROSOFT. It monitors the patients SPo2, HR, location information for automatic detection of sleep apnea events. The authors reported the results from a sleep study on 20 participants, which validated the feasibility of the concepts and solutions adopted by the project. 
All these studies disclosed that, the discussed Mobile health care systems have great potential for continuous monitoring, and early detection of abnormal conditions.

These systems can provide patients with increased confidence and a better quality life. Researches must be undertaken to design communication protocols, efficient energymanagement schemes, and encryption algorithms. Right hardware components for specific applications should be selected carefully. In addition to these technical issues, economics of operating a mobile healthcare system also need to be analyzed carefully to ensure emergency and preventive healthcare monitoring is affordable to all levels of society [8] [9].

The fifth research is done by Hariharasudhan Viswanathan, et al. [10]. The authors introduced a prediction of ubiquitous healthcare systems future. It will be characterized by:

- Pervasive vital sign monitoring using noninvasive sensors.

- Real-time processing of monitored data to derive meaningful physiological parameters.

- Context-aware data and patient-centric decision making.

The research proposed an autonomic resource provisioning framework that can utilize every available computing resource in the vicinity to support both real-time processing of vital signs acquire context awareness, using inherently complex physiological models and machine-learning-based algorithms. Fig. 6 shows concept diagram illustrating the envisioned ubiquitous healthcare solution introduced by Hariharasudhan Viswanathan, et al. [10].

Contextual information such as physical activity, location, and environmental conditions can be obtained by processing data from kinematic sensors (accelerometers and gyroscopes), GPS, and temperature/humidity sensors. Correlation of derived physiological parameters with contextual information can be used for automated diagnosis of physiological conditions.

For example, looking into the cardiac health of a patient can be acquired by correlating the HR, BP with contextual information such as state of exertion or rest identified using physical activity recognition, which can be effective in recognition of the appropriate response and medical treatment required in case of sport persons, or elderly.

The introduced system is mainly working in offline mode where the collected data can be locally stored on the sensor nodes and can be analyzed later using powerful processing devices such as a desktop computer in a delay-tolerant fashion.

Alerts and reports of early diagnosis can be made available to health service provider through a remote online electronic health record database (not in real-time).

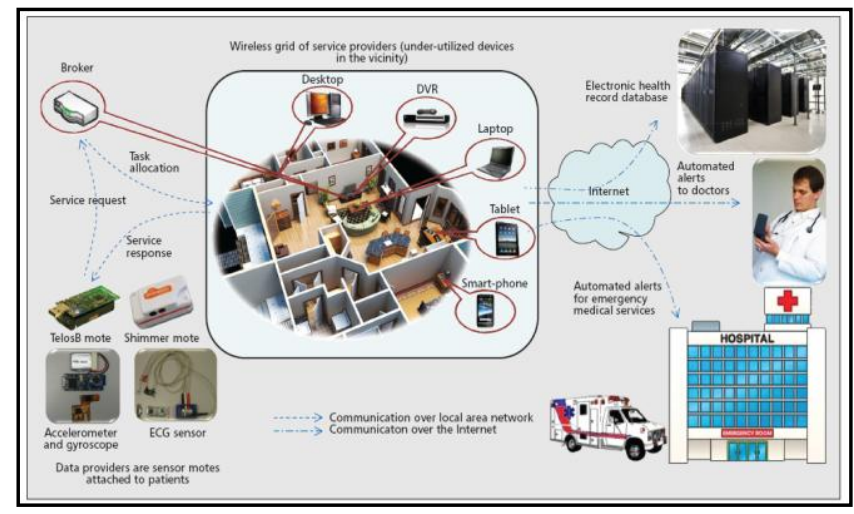

Fig 6: Concept diagram illustrating the ubiquitous health care solution [10]

The proposed system is not suitable in case of emergency situation as the data collection and processing is carried out in offline mode.

\section{REQUIREMENTS OF PERVASIVE HEALTH CARE SYSTEMS}

Pervasive health care applications can be categorized as follows: health care prevention, health care checkups and maintenance, short-term monitoring, long-term monitoring, personalized health care services, emergency detection, emergency intervention, patient positioning and transportation, and finally treatment services [6] [11].

Referring to all the previously reviewed ubiquitous health care systems and categories general requirements can be summarized as follows [1] [2] [6] [11]:

- Highly reliable and usable middleware devices to ensure the maximum levels of system's functionality.

- Highly reliable and usable wireless infrastructure that is available all the time even by keeping redundant resources to ensure the required connectivity. It should also support prioritized communications.

- High level of security to prevent attacks and provide protection of confidentiality, integration and availability.

- High level of privacy should be provided, where sensitive patients' data will not be compromised even if the health care system is attacked. This can be done by encrypting patients' data.

- Costly Affordable and user friendly interfaces. It should be non-intrusive.

- Autonomous and context-aware responses should be considered in the system design and implementation.

- The system should be designed to provide patient's location and support tracking facility even if the patient is mobile or in rural area.

- Improvement in the correctness of personalized medical decisions making in correspondence to the context awareness inputs as well as the physical inputs should be granted.

- The system should digest all requiring patients and be scalable to assimilate more.

\section{PROPOSED SOLUTION}

As mentioned that, continuous advances of medical technologies and methodologies, helped in the noticeable extension of human average living age, which in turn increased the pressure of aging population on healthcare systems. Also increased the financial support afforded by governmental organization to comply with the requiring health care insurance sector. So the authors are trying to develop a solution for emergency management in developing countries especially for elderly un-capable patients with chronic heart diseases. This solution is affordable and can be adopted by governmental health care organization to help cardio-patients getting proper treatment on time (Aff-HRM)

\subsection{SYSTEM DDESCRIPTION AND SPECIFICATIONS:}

Ubiquitous computing and IoT principles and technologies have been widely used in health care services' improvement. 
Also huge advancements in smart mobile devices and their application make it the most applicable device to be used in health care interventions because of; its wide spread even among financially un-capable persons, people's attachment to their mobile devices and their tendency to carry them everywhere, and finally the easiness of using mobile phones and context-awareness features embedded in them that gives the feeling that smart mobile phone understands human needs.

Some of smart phones technical features that made their intervention in health care domain much easier are, text messaging (SMS), embedded cameras, programmable feature (any application can be customized to be installed on the smart phone environment), automated sensing features (Bluetooth, PAN), and finally their internet connectivity features [12].

- Aff-HRM components are described as follows:

- HBM sensor: Bluetooth-enabled sensor is preferable as it is unlikely to ANT-enabled sensors. Bluetooth is one of the most popular embedded services in all mobile handheld devices. Also it has a low power consumption level compared to WLAN enabled sensors. This sensor is a mainly a wearable strap, that is positioned just below the patient's chest. It measures the patient's heart beat rate and it can draw the heart ECO signal using the accompanying application.

- Windows/android based mobile device: Bluetooth/WLAN/GPS/GSM enabled mobile device is representing the main interface between the HBM and health care service provider. The smart phone should receive the $\mathrm{HB}$ rate signals via Bluetooth and update its local application database which in turn is communicating with the service provider web service to update the patient's electronic medical record via internet connection WLAN or GSM. Smart phone application is managing three types of alerting processes; SMS alert to any relative or neighbor of the monitored patient, SMS alert sending the determined positioning and locating of the patient via GSM/GPS, and finally update the health care service provider data base with the current status of the patient and issuing an alarm when the monitored HB rate is exceeding certain value which in turn is alerting the emergency unit in the hospital to send an ambulance with the trained medics and equipments needed to rescue the suffering patient.

- Server Application: This is responsible for the data reception and analysis. It is responsible for decision making according to the received data.

The server should be connected with the emergency center and ambulance systems. The server should react with the incident in a timely manner to rescue the suffering patient.

Aff-HRM implementation is based on service oriented architecture (SOA). The whole Aff-HRM system structure is shown in Fig. 7.

A prototype of Aff-HRM has been implemented to test its functionality and feasibility. Screen shots of different system components are shown in Fig. 8 and Fig. 9

Currently Aff-HRM prototype is applied to different patients in different environments. It is in testing phase but till now it is giving the expected response which confirms the feasibility of the system.

\section{MITIGATED CHALLENGES IN HEALTHCARE DOMAIN}

Several factors are affecting the development of health care industry; these factors are widely mitigated by using

\section{- Global challenges in healthcare}

○ Chronic and infectious diseases

$\circ \quad$ Ageing populations

○ Urbanization and environmental impact

$\circ$ Rising costs of healthcare

- Prevention of life threatening medical incidents

pervasive health care models as previously presented in the ubiquitous health care features and capabilities [13].

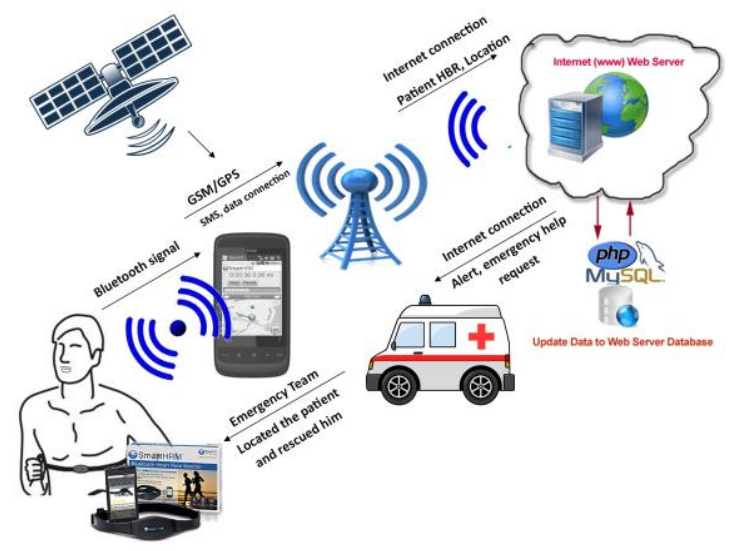

Fig 7: Aff-HRM Architecture

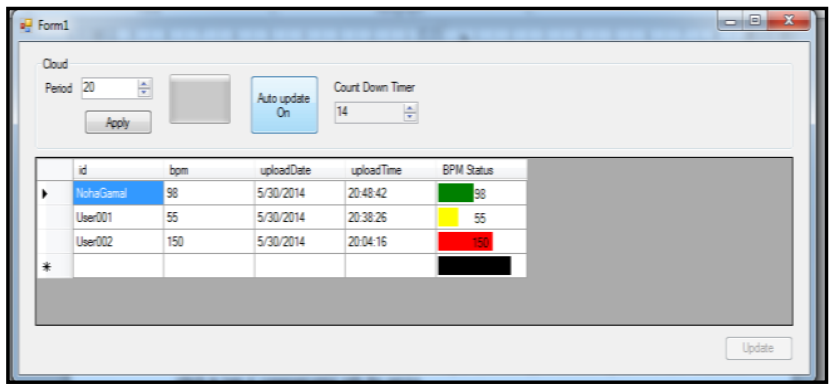

Fig 8: Client Side Application Receiving HBR from HBM via Bluetooth.

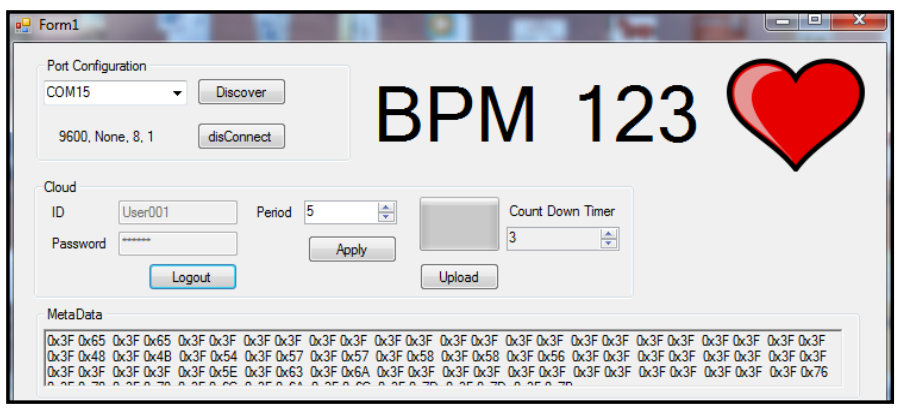

Fig 9: Server Side Application Receiving HBR from Mobile Client via GPRS 


\section{CONCLUSION AND FUTURE WORK}

Pervasive health care is used to shift the already established, hospital based, centralized health care system to another ubiquitous, user-centered, and mobile system as an overall preventive health management system. In ubiquitous health care there is a seamless integration between academic researches and advances in industry, which results in rapid adoption of academic researches outcomes in industry or wide range of available industrial schemes to support academic researches.

The main features and requirements that should be provided by a reliable pervasive health care system; those are: Uses highly reliable and usable middleware; existing highly reliable and usable wireless infrastructure; high level of security to prevent attacks; high level of privacy should be provided; costly affordable and user friendly interfaces; it should be non-intrusive; Autonomous and context-aware responses should be considered; support tracking facility of the patient even if he is mobile or in rural area; also the system should digest all requiring patients and to be scalable to assimilate more; and finally Improvement in the correctness of personalized medical decisions in correspondence to the context awareness inputs as well as the physical inputs should be granted.

The proposed solution of real-time cardiovascular emergency detection and response system is affordable and can be adopted be governmental health care organization to help cardio-patients getting proper treatment on time (Aff-HRM). Aff-HRM implementation is based on service oriented architecture (SOA); using Bluetooth-enabled sensor to measure the patient's heart beat rate and it can draw the heart ECO signal using the accompanying application. Windows/android based Bluetooth/WLAN/GPS/GSM enabled mobile device is representing the main interface between the patient and health care service provider.

Aff-HRM prototype is currently applied to different patients in different environments and it is in testing phase but till now it is giving the expected response which confirms the feasibility of the system.

Now it is obvious that several factors are affecting the development of health care industry; these factors are widely mitigated by using pervasive health care models as previously presented in the ubiquitous health care features and capabilities.

Author is planning to extend the proposed solution to include measuring other human vital signals that can indicate more critical emergency situations like, falling detection for elderly people, blood pressure, body temperature, and glucose levels. That to extend the capabilities of the proposed system while keeping the main features of being costly affordable and user friendly.

\section{REFERENCES}

[1] A Mukhopadhyay S., A Postolache, O.A., "Pervasive and Mobile Sensing and Computing for Healthcare: Technological and Social Issues,”2012, Springer.
[2] Cacciagrano, D., Corradini, F., Culmone, R., Merelli, E., \& Vito, L. "Healthcare Tomorrow: Toward Selfadaptive, Ubiquitous and Personalized Services," UBICOMM 2011, The Fifth International Conference on Mobile Ubiquitous Computing, Systems, Services and Technologies (pp. 245-250) (2011, November).

[3] Rastegari, Elham, Amirmasood Rahmani, and Saeed Setayeshi. "Pervasive Computing in Healthcare Systems, " World Academy of Science, Engineering and Technology 59 (2011)

[4] Orwat, Carsten, Andreas Graefe, and Timm Faulwasser. "Towards pervasive computing in health care-A literature review," BMC Medical Informatics and Decision Making 8.1 (2008): 26.

[5] Moon, Junho, and Dongsoo Kim. "Context-Aware Business Process Management for Personalized Healthcare Services," In Services Computing (SCC), 2013 IEEE International Conference on, pp. 757-758. IEEE, 2013

[6] Crosby, Garth V., et al. "Wireless Body Area Networks for Healthcare: A Survey," International Journal of Ad Hoc, Sensor \& Ubiquitous Computing 3.3 (2012).

[7] S. S. Marci, et al., "Security and Privacy Issues with Health Care Information Technology," in 28th Annual International Conference of the IEEE Engineering in Medicine and Biology Society (EMBS 06), New York City, NY, USA, 2006, pp. 5453-5458.

[8] Bhadoria, Sanjay S., and Hitesh Gupta. "A Wearable Personal Healthcare and Emergency Information Based On Mobile Application," ISROSET- IJSRCSE Vol-1, Issue-4, PP (24-30) July-August 2013 (2013).

[9] Shahram Jalaliniya, Thomas Pederson, "A wearable kids' health monitoring system on smartphone," In 7th Nordic Conference on Human-Computer Interaction: Making Sense Through Design, 2012, pp.791-792.

[10] Viswanathan, Hariharasudhan, Baozhi Chen, and Dario Pompili. "Research challenges in computation, communication, and context awareness for ubiquitous healthcare," Communications Magazine, IEEE 50.5 (2012): 92-99.

[11] Varshney, Upkar. "Pervasive healthcare and wireless health monitoring," Mobile Networks and Applications 12.2-3 (2007): 113-127.

[12] Klasnja, Predrag, and Wanda Pratt. "Healthcare in the pocket: Mapping the space of mobile-phone health interventions," Journal of biomedical informatics 45.1 (2012): 184-198.

[13] Atallah, Louis, Benny Lo, and Guang-Zhong Yang. "Can pervasive sensing address current challenges in global healthcare?," Journal of epidemiology and global health 2.1 (2012): 1-13. 